\title{
Turisti a Lampedusa. Note sul nesso tra mobilità e patrimonio nel Mediterraneo
}

Tourists in Lampedusa. Notes on the relationship between mobility and heritage in the Mediterranean

\section{Francesco Vietti}

\section{(2) OpenEdition}

\section{Journals}

Edizione digitale

URL: http://journals.openedition.org/aam/1252

DOI: $10.4000 /$ aam. 1252

ISSN: 2038-3215

\section{Editore}

Dipartimento Culture e Società - Università di Palermo

Notizia bibliografica digitale

Francesco Vietti, «Turisti a Lampedusa. Note sul nesso tra mobilità e patrimonio nel Mediterraneo », Archivio antropologico mediterraneo [Online], Anno XXII, n. 21 (1) | 2019, online dal 30 juin 2019, consultato il 23 septembre 2019. URL : http://journals.openedition.org/aam/1252 ; DOI : 10.4000/ aam. 1252

Questo documento è stato generato automaticamente il 23 septembre 2019

Archivio antropologico mediterraneo 


\section{Turisti a Lampedusa. Note sul nesso tra mobilità e patrimonio nel Mediterraneo}

Tourists in Lampedusa. Notes on the relationship between mobility and heritage in the Mediterranean

\section{Francesco Vietti}

\section{Introduzione}

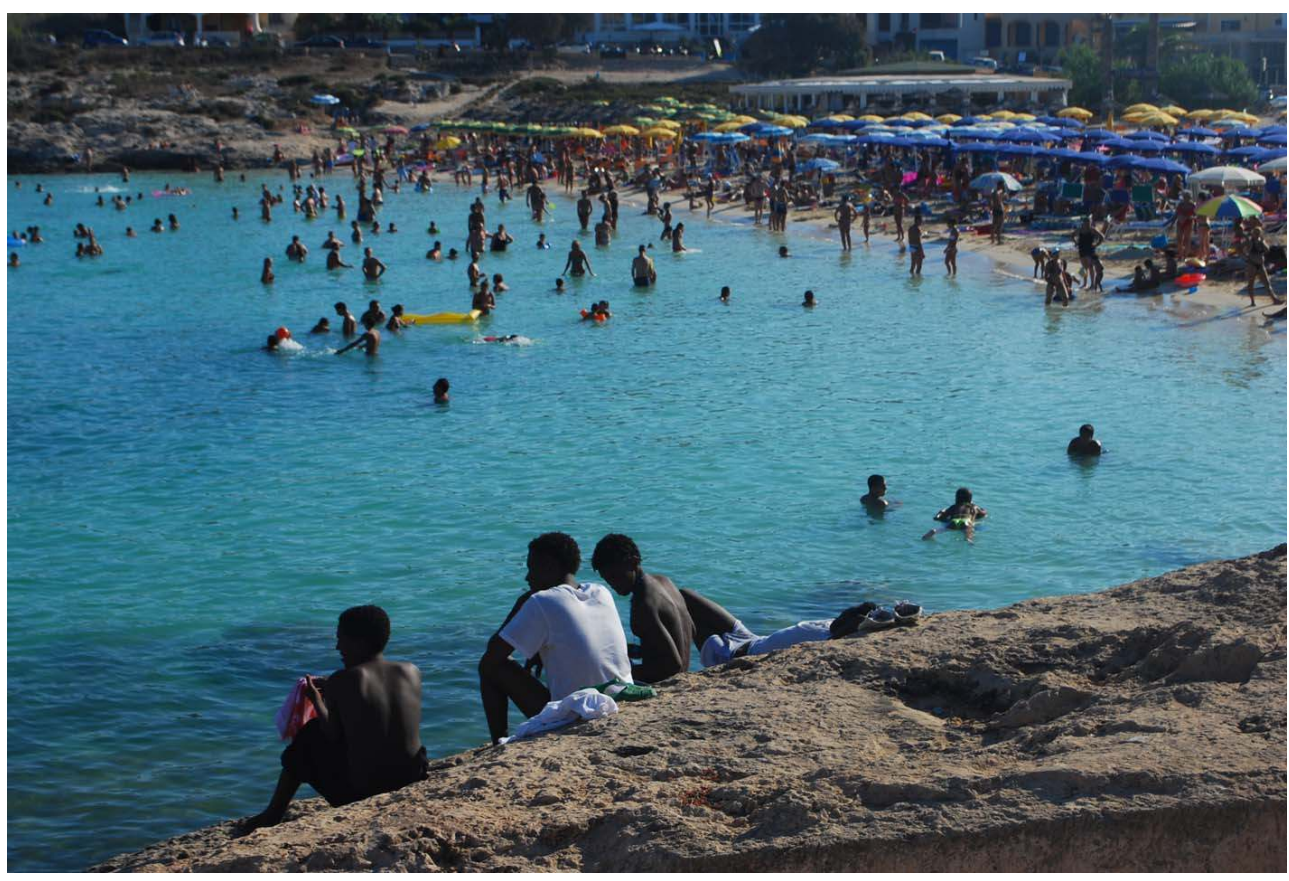

1 Ho scattato la fotografia qui riprodotta il 25 luglio 2013. Era la prima volta che mi trovavo a Lampedusa e vi arrivai come turista. Occupandomi da alcuni anni di 
migrazioni, come molte altre persone avevo letto reportage e saggi su Lampedusa, l'avevo vista in televisione, l'avevo immaginata ascoltando i racconti di chi, transitato dall'isola, era poi giunto in altre parti d'Italia attendendo l'esito della sua richiesta d'asilo.

2 Nel luglio del 2013 sull'isola co-abitavano alcune migliaia di turisti intenti a divertirsi e rilassarsi, diverse centinaia di migranti impegnati in una lotta contro la pratica della raccolta forzosa delle impronte digitali e decine di studiosi, giornalisti, attivisti e artisti variamente interessanti a indagare, testimoniare, rappresentare e rielaborare quanto stava accadendo nel Mediterraneo. Oltre ovviamente ai circa 6.000 abitanti del posto.

3 L'immagine mi sembra cogliere uno degli aspetti di quanto notai durante il primo soggiorno a Lampedusa. Lo scatto inquadra la spiaggia della Guitgia, una delle più frequentate e comode rispetto al centro di Lampedusa paese. Sullo sfondo si nota un gran numero di turisti e una selva di ombrelloni. In primo piano, seduti sugli scogli che delimitano la spiaggia, vi sono invece alcuni giovani migranti del Corno D'Africa, intenti a osservare il mare. Un "incontro mancato" (Aime 2005) che mi ha molto interrogato negli anni seguenti, spingendomi a un duplice interesse teorico e applicativo: da quel momento in avanti sono tornato ogni anno sull'isola, ricoprendo di volta in volta diversi ruoli (ricercatore di antropologia, accompagnatore di turismo responsabile, tutor per un progetto di alternanza scuola-lavoro del locale istituto turistico-alberghiero) ${ }^{1}$.

4 Nel presente articolo intendo indagare il complesso immaginario turistico relativo a Lampedusa, un'isola che si è storicamente connotata per la sua estrema marginalità nei confronti del territorio italiano, e al tempo stesso per la sua centralità rispetto allo spazio mediterraneo compreso tra la Sicilia, Malta e la Tunisia. Mi propongo di tracciare un profilo della storia dell'isola, con particolare riferimento al ruolo svolto dalle diverse forme di mobilità che l'hanno caratterizzata nelle successive fasi di trasformazione sociale, politica ed economica. Mi concentrerò in particolare sui processi di patrimonializzazione in atto, chiedendomi se e come le migrazioni contemporanee siano state integrate nel patrimonio culturale materiale e immateriale di Lampedusa: a tal fine analizzerò alcune dimensioni di ri-significazione simbolica delle celebrazioni per la Festa della Madonna di Porto Salvo, patrona dell'isola e dei suoi pescatori, divenuta recentemente protettrice di turisti e migranti.

5 Per sviluppare le mie argomentazioni farò ricorso a un corpus di interviste e annotazioni etnografiche raccolte tra il 2013 e il 2018 durante cinque periodi di permanenza a Lampedusa, nonché alla documentazione reperita grazie alla collaborazione con l'Archivio Storico di Lampedusa e altre associazioni locali ${ }^{2}$.

\section{La mobilità e i suoi confini}

Per cominciare a decifrare la complessità dello scenario di Lampedusa, mi sembra utile evocare, ad oltre vent'anni dalla sua formulazione, il noto concetto di ethnoscape, coniato da Arjun Appadurai per individuare uno dei flussi culturali globali della contemporaneità. È proprio nei panorami della globalizzazione che, secondo l'antropologo indiano, «turisti, immigrati, rifugiati, esiliati, lavoratori ospiti, ed altri gruppi e individui in movimento» si trovano fianco a fianco e influenzano «la politica delle (e tra le) nazioni» (Appadurai 2001 [1996]: 53). Un accostamento che trova riscontro anche nella coeva definizione della cultura-come-rapporti-di-viaggio proposta 
da James Clifford, da cui discende la necessità di realizzare delle etnografie dei siti di transito attraversati da turisti, viaggiatori, etnografi, funzionari governativi, poliziotti, mercanti, pellegrini, artisti, lavoratori stagionali e immigrati, nonché da «potenti forze che passano attraverso», come la televisione, le merci, gli eserciti (Clifford 2008 [1997]: 39).

7 Come ha recentemente ricostruito Bruno Riccio (2019), l'attenzione rivolta alla comune opera dell'immaginazione nei processi di rappresentazione dell'altro e dell'altrove ha permesso di connettere tradizioni di studio precedentemente autonome: da un lato le ricerche sulle diverse scale della migrazione (interna, internazionale, transnazionale), dall'altro quelle sul viaggio e sul turismo. Emblematico in tal senso è stato il lavoro di John Urry (2000), forse il maggior artefice di quello che sarebbe stato definito mobility turn o new mobilities paradigm (Adey 2017). A partire dagli anni Duemila, la migrazione e il turismo sono dunque entrati sempre più spesso a far parte di un più ampio ambito di indagine sulle dimensioni della mobilità umana e sul suo impatto sulla trasformazione dei luoghi.

8 Sono così caduti alcuni confini tra ambiti della mobilità che hanno permesso di disarticolare la consolidata contrapposizione tra produzione e consumo come elementi connotanti in modo esclusivo rispettivamente l'esperienza della migrazione e del turismo, e di esplorare le diverse declinazioni del migration-tourism nexus (Hall, Williams 2002). Nell'area mediterranea, particolarmente interessante è stato l'approccio comparativo con cui Ramona Lenz (2010) ha messo in discussione la tradizionale dicotomia host-guest a Cipro e Creta. In entrambi i casi, il luogo prescelto dall'antropologa tedesca per condurre le proprie osservazioni è stato l'hotel, cronotopo per eccellenza dell'esperienza turistica, divenuto negli ultimi anni in molti contesti mediterranei anche spazio di vita, lavoro, transito o accoglienza per diverse generazioni di migranti.

Muoversi su questo campo ibrido, fatto di connessioni e sovrapposizioni, non significa tuttavia trascurare o sottovalutare le profonde differenze tra "regimi di mobilità" (Glick Schiller, Salazar 2011) che permangono tra turisti e migranti, e che molto spesso vengono anzi rafforzate dai dispositivi di controllo, sorveglianza e governo della libertà di movimento nel mondo neo-liberale. Nina Glick-Schiller e Noel Salazar hanno indicato chiaramente le necessità di non de-politicizzare il tema, mantenendo al contrario elevata l'attenzione sulle gerarchie di status e sulle condizioni di diseguaglianza ed esclusione prodotte dalle possibilità e dalle costrizioni legate all'inscindibile binomio mobilità/immobilità. Siamo dunque di fronte a una linea di demarazione che unisce e al tempo stesso divide i vari soggetti in campo, delimitando l'area di una vasta area di frontiera in cui rientrano questioni identitarie, asimmetrie di potere, scambi interculturali. Introducendo il volume collettaneo Border Encounters Asymmetry and Proximity at Europe's Frontiers, Jutta Lauth Bacas e William Kavanagh (2013) hanno scritto a tal proposito:

Since frontiers bring people together in spatial proximity (though clearly such physical proximity does not necessarily entail social proximity), (...) of great interest are the hierarchical relations between the people who meet at international borders: permanent residents on one or another side of an international frontier, as well as migrants, travellers, tourists, traders and pensioners in interaction with border guards, police officers or security personnel with the power to grant or to delay passage beyond the physical limit of the frontier (Lauth Bacas, Kavanagh 2013: 2). 

inquadrato l'incontro di frontiera che qui avviene tra i migranti provenienti dal Medio Oriente, gli abitanti locali, i numerosi turisti europei, le forze di polizia e gli operaotori delle organizzazioni non governitve presenti sull'isola. Nelle strutture di prima accoglienza e nello spazio pubblico del principale centro urbano e turistico dell'isola, la cittadina di Mytilene, è possibile osservare chiaramente i processi attraverso cui si sedimentano gli immaginari sulla reciproca alterità e si costruiscono relazioni gerarchicamente strutturate, in cui centrale è la gestione della prossimità/distanza tra i diversi attori e l'elaborazione della dimensione del confine come repertorio di discorsi, di norme e di pratiche (Lauth Bacas 2013).

11 Il caso delle isole del Mar Egeo tra Grecia e Turchia può essere richiamato per un diretto confronto con il contesto lampedusano. Anche qui ci si trova infatti su un tratto della frontiera europea, o meglio, sul palcoscenico dove ormai da circa un ventennio si mette in scena quello "spettacolo del confine" svelato dall'ormai classico studio di Paolo Cuttitta (2012). Una rappresentazione che ha alternato nel corso del tempo le retoriche dell'accoglienza, con l'emblema della "Porta d'Italia - Porta d'Europa" eretta in ricordo delle vittime delle traversate mediterranee, e quelle dei respingimenti, con lo slogan dei "porti chiusi" e le navi cariche di migranti lasciate in mare di fronte alle coste dell'isola. Immagini apparentemente contrapposte, eppure complementari nell'articolare gli slittamenti tra il discorso securitario e quello umanitario che ormai da lungo tempo costituiscono i due poli della gestione dei flussi migratori tra le sponde del Mediterraneo. Un'ambivalenza che si riverbera nelle stratificazioni di immaginari con i quali i turisti giungono sull'isola.

Ed eccoci così tornati sulla spiaggia della Guitgia. Quel giorno dell'estate 2013, conversando con i turisti distesi sulle sdraio accanto al mio ombrellone, raccolsi lo sfogo di Marilena, pensionata milanese di 63 anni, che indicando con la mano i migranti seduti sugli scogli mi disse:

E dire che gliel'avevo chiesto a quelli dell'agenzia se li avavano portati via tutti i clandestini, e loro mi avevano garantito che non li avrei visti i negri quest'anno. Non riesco a rilassarmi, a godermi la vacanza se c'è tutta questa gente in giro per il paese, non so come faccia la gente di qui a sopportare questa situazione da tanti anni!

Su quella stessa spiaggia, due anni più tardi, sarei tornato accompagnando un gruppo di turisti nell'ambito di un soggiorno di turismo responsabile. E avrei ascoltato da Carla, trentenne di Pinerolo, una recriminazione di segno opposto:

Io mi sono iscritta perché sono interessata alla questione delle migrazioni, lo sai no, te l'ho detto dal primo giorno, ma qui di migranti non se ne vedono, ce ne sono molti di più a Torino, per dire. Secondo te non riusciremo a parlare con qualche ragazzo sbarcato ad esempio, o visitare il centro di accoglienza? Io ci terrei ad assistere a uno sbarco...

14

In entrambi i casi, l'espressione di una delusione relativa al modo con cui lo "sguardo turistico" vede la presenza/assenza dei migranti a Lampedusa: sempre troppo vicini o troppo lontani, mai alla giusta distanza.

Archivio antropologico mediterraneo, Anno XXII, n. 21 (1) | 2019 


\section{Hotel Lampedusa}

15 Lampedusa è più prossima alla costa tunisina che a quella siciliana. A Zarzis, cittadina non distante da Djerba, si trovano diversi alberghi e ristoranti intitolati all'isola italiana. Come mi ha spiegato Lassaad, proprietario del "Restaurant Lampedusa" nella via principale della città:

Quando ho aperto l'attività sapevo che questo era il nome migliore da dare. Ai turisti italiani fa sempre piacere leggere un nome del loro paese, li fa sentire a casa. E per quanto riguarda $i$ tunisini, tutti qui sono legati a Lampedusa... c'è chi ha lavorato sulle navi da pesca con i lampedusani e tutti hanno qualche amico e parente che ci è passato viaggiando verso l'Europa.

Le immagini e i racconti che circolano in Tunisia restituiscono un riflesso dell'importante ruolo che le diverse forme di mobilità hanno avuto per Lampedusa nel corso del tempo. Ed è questo il punto che vorrei ora mettere a fuoco, rileggendo alcune fasi significative della sua storia sociale ed economica attraverso la prospettiva del rapporto tra migrazioni, turismo e processi di patrimonializzazione ${ }^{3}$.

\subsection{Coloni e dammusi}

17 Il punto di partenza per questa mia esplorazione etnografica non può che essere il centro di Lampedusa paese, all'incrocio tra i due principali assi viari dell'abitato, via Roma e via Vittorio Emanuele. Qui si trova infatti una delle tracce più evidenti della colonizzazione dell'isola, ossia del processo di insediamento che ha avviato la storia moderna di Lampedusa. I turisti che tutte le sere passeggiano tra i negozi e le bancarelle di via Roma, di fronte all'edificio che ospita il Comune di Lampedusa e Linosa trovano un pannello che recita:

Nel 1844, l'anno successivo alla colonizzazione di Lampedusa, in Sicilia fu bandito l'appalto per la costruzione delle case coloniche [...]. Da Palermo giunsero a Lampedusa molti brigantini degli appaltanti per trasportare sull'isola architetti, capimastri e più di 500 operai edili, falegnami, tagliapietre, fornaciai, carrettieri muniti di carri e cavalli; di tanto in tanto arrivava il materiale edile occorrente per le nuove costruzioni. Gli operai portarono con loro le famiglie e alloggiarono in baracche di legno costruite alla svelta. Il piano di "fabbricazione" del paese fu redatto e successivamente [...] l'architetto D. Nicolò Puglia progettò le case che sorsero allineate lungo una linea parallela al porto in numero di sette edifici isolati aventi ciascuno dieci abitazioni.

18 I sette palazzi che sancirono la fondazione della colonia borbonica sono tuttora esistenti, presentano un difforme stato di conservazione e differenti destinazioni d'uso: alcuni sono adibiti ad abitazione privata, altri ospitano al pian terreno negozi e ristoranti, uno è sede di Legambiente.

Il popolamento di Lampedusa avvenne in breve tempo tramite il trasferimento di persone provenienti da altri territori del Regno Borbonico, da isole come Pantelleria e Ustica e dalle aree di Agrigento e Palermo. Secondo i piani iniziali, la colonia avrebbe dovuto avere vocazione agricola, dunque ai coloni furono assegnate porzioni di terreno per la coltivazione. Qui vennero costruite abitazioni in pietra, chiamate dammusi, modellate sulla tipologia abitativa rurale tipica del Mediterraneo centrale. A distanza di un secolo e mezzo, sono proprio queste case coloniche ottocentesche a svolgere un ruolo centrale nel discorso relativo a ciò che di "autentico" l'isola ha da offrire ai 
visitatori. Il processo di patrimonializzazione è stato duplice: da un lato le istituzioni hanno promosso la musealizzazione di uno dei dammusi storici, risalente al 1870, ristrutturato e aperto al pubblico nel 2006; dall'altro, gli imprenditori locali hanno investito nella costruzione di centinaia di nuovi dammusi per alloggiare i turisti interessati a una sistemazione "tradizionale". Emblematico in tal senso è il caso dei "Dammusi di Borgo Cala Creta", il primo e tuttora più esteso villaggio turistico di Lampedusa, ideato ed edificato non senza polemiche e scontri giudiziari negli anni '70 del secolo corso. Racconta Annalisa, una delle imprenditrici che ha reinventato i dammusi lampedusani come case-vacanza:

Quando sono arrivata qui per la prima volta nel 1969 non c'era nulla, neanche un hotel, solo una pensione molto mal messa nella zona dello sbarcatoio. Ero venuta qui in vacanza da Firenze, dove ero proprietaria di un negozio di abbigliamento in centro, di grande successo. Mi innamorai subito dei colori dell'isola, delle sue pietre, con il contrasto cromatico con i fichi d'india e il mare. Le case moderne che si costruivano allora in cemento erano brutte, rovinavano l'estetica dell'isola e così io pensai di fare qualcosa di bello, quelli erano gli anni in cui nasceva il Club Méditerranée e io progettai con i miei soci qualcosa di simile qui a Lampedusa.

\subsection{Spugne e pescatori}

L'iniziale sviluppo agricolo della colonia borbonica fu ben presto soppiantato dall'emergere del dominante settore della pesca. Nel $1887 \mathrm{fu}$ infatti scoperto non lontano dalle coste dell'isola un grande banco di spugne di mare, che in breve tempo attirò a Lampedusa una moltitudine di pescatori provenienti da Tunisia, Grecia, Dalmazia e Turchia. I lampedusani divennero così in buona parte "spugnari" e l'isola si trasformò nel centro di un fiorente commercio mediterraneo che portò a un significativo miglioramento delle condizioni di vita sull'isola. Di quest'antica attività e delle relativa immigrazione che l'accompagnò è testimone uno dei negozi più frequentati dai turisti lungo via Roma. Si tratta dello "Spugnificio Giovannino", l'ultimo rimasto in attività a Lampedusa: non un semplice emporio dove acquistare souvenirs, ma un piccolo museo dedicato a un aspetto del patrimonio materiale dell'isola prodotto da un'antica forma di mobilità lavorativa. Il proprietario, Calogero, ama intrattenere i visitatori con le sue storie di famiglia:
Mio nonno era greco, è lui che è venuto a Lampedusa e ha cominciato l'attività. Le spugne si pescavano con una barca chiamata saccalleva e con lo scafando da palombaro. Una vita durissima figurati... una volta il Mediterraneo era più unito, si circolava più liberamente, ora con tutte queste regole delle acque territoriali è un casino. Ora non si fa più la pesca della spugna, le spugne vengono raccolte solo con la rete a strascico, come materiali diciamo residuale. Comunque le nostre spugne sono tutte naturali, lavorate a mano, senza sbiancanti chimici, solo che queste cose le devi spiegare ai turisti che vengono qui, perché non le sanno, non sanno distinguere le spugne naturali da quelle industriali.

21 L"'epoca d'oro" della pesca alle spugne durò alcuni decenni, ma fin dai primi del Novecento si affermarono anche altre tipologie di pesca, a partire da quella del pesce azzurro. Lampedusa divenne così per la prima metà del secolo "l'isola degli sgombri" (Roghi 2013): una pesca fortemente stagionalizzata, praticata tra maggio e settembre, periodo del passaggio dei banchi di sgombri nel braccio di mare tra Lampedusa e la Tunisia, e capace di impiegare la quasi totalità degli uomini in mare e delle donne sulla terraferma. Sorsero infatti sull'isola numerose ditte per la lavorazione del pescato, una delle quali, la "Ditta Famularo", è attiva ancor oggi e con i suoi diversi negozi tra le vie 
del paese costituisce la principale attrazione per i turisti in cerca di souvenirs gastronomici.

Come si evince rileggendo a distanza di qualche anno il pionieristico studio su cultura orale e analfabetismo a Lampedusa condotto da Matilde Callari Galli e Gualtiero Harrison (1972), il secondo dopoguerra fu un'epoca in cui l'isolamento di Lampedusa fu particolarmente severo e l'emigrazione divenne massiccia: si vennero così a creare numerose comunità di lampedusani fuori dall'isola, sia in Italia (particolarmente numerose ad Anzio, Ancona, Rimini, Viareggio) che all'estero. Le condizioni sull'isola risentivano della mancanza dei servizi di base: fino agli anni '60 mancavano rete fognaria, presidio medico e scuole e i collegamenti con la Sicilia erano garantiti da una nave che giungeva alle Pelagie due sole volte a settimana. La protesta degli isolani, che nel 1966 decisero di disertare collettivamente le urne, impose all'allora Ministro dell'Interno Taviani di recarsi sull'isola per avviare una serie di interventi che in breve tempo l'avrebbero trasformata: nel 1967 fu portata la prima linea telefonica e nel 1968 fu realizzato l'aeroporto civile e messo in funzione il dissalatore per l'acqua marina (Taranto 2013).

Nel frattempo molti giovani uomini lampedusani, in cerca di migliori compensi, avevano preso a dedicarsi alla "pesca atlantica": si imbarcavano su grandi pescherecci per la pesca d'altura nell'oceano e rimanevano lontani da Lampedusa per una buona parte dell'anno. Fu una mobilità per lavoro di ampio raggio, che mise per la prima volta un'intera generazione a contatto con u' Marroccu, ossia il mondo oltre lo Stretto di Gibilterra. Un periodo che ricorre spesso nella memoria degli ex-pescatori che negli ultimi decenni hanno abbandonato il mestiere per dedicarsi ad un altro genere di occupazione marinara: portare i turisti a fare il giro in barca dell'isola e accompagnarli nelle immersioni subacquee. Tra le decine di marinai che al porto di Lampedusa offrono questo servizio ai visitatori, vi è anche "Franciscu il pescatore", come recita il suo biglietto da visita:

Son 24 anni ormai che porto i turisti, lavoro sei mesi all'anno, non mi lamento, ma d'estate non c'è festa e non c'è domenica, si lavora tutti i giorni. Io ho clienti fissi, che vengono con me tutti gli anni, e ci sono anche quelli nuovi, come i giapponesi e i cinesi, che son sono arrivati da poco. Io i soldi e la casa, come tutti qui, me li sono fatti a colpi di mare in faccia, come si dice... ho navigato per anni, facevo campagne anche di sei mesi, era dura, e bisognava convivere con tutti a bordo, con noi lampedusani c'era gente di altri paesi, tunisini, somali... Sono stato ovunque, Marocco, Triangolo delle Bermuda, New York, Canada. Che tempi... ora invece sono tranquillo, ho la mia barchetta, porto dodici persone alla volta, gli faccio fare il giro di tutte le spiagge e faccio anche la grigliata di pesce fresco a bordo, sono tutti contenti, basta che guardi la mia pagina Facebook.

\subsection{Missili e turisti}

All'inizio degli anni '70 si posero dunque le condizioni perché l'isola potesse cominciare ad attirare e accogliere visitatori italiani e stranieri. Parallelamente ai primi turisti giunse a Lampedusa un altro gruppo di "popolazione mobile" che non avrebbe più abbandonato l'isola: i militari. Nel 1972, sulla punta occidentale dell'isola, venne infatti installata una base radar di tipo "Loran" gestita per conto della NATO da un contingente di soldati statunitensi. Proprio a questa base erano i destinati i famosi missili che il colonnello libico Muhammar Gheddafi lanciò nel 1986 per ritorsione al bombardamento americano di Tripoli. Come la mitologia turistica isolana non esita a 
rimarcare, sarebbe stata la visibilità mediatica dovuta ai "siluri di Gheddafi" a far la fortuna di Lampedusa, propiziando il definitivo passaggio dell'isola dall'economia della pesca a quella del turismo (Isola 2018).

Spinti anche dalle nuove e più restrittive regole del mercato unico europeo nel settore ittico, i pescatori dismisero i loro pescherecci e investirono i loro capitali nella costruzione di case da affittare ai villeggianti, nonché di alberghi, ristoranti e attività commerciali rivolte principalmente agli ospiti delle stagione balneare (Orsini 2015). L'afflusso di migliaia di turisti, una popolazione ben più numerosa di quella residenti sull'isola, ebbe un tangibile impatto sulla società e sull'economica locale. $\mathrm{Ne}$ "Le isole del sole", la prima guida turistica delle Pelagie, Enzo Macini così coglieva le ambivalenze e le tensioni, anche intergenerazionali, di quel peculiare momento di passaggio:

Si sta verificando una situazione di squilibrio e persino di caos nel periodo di punta (agosto), quando più massiccia è la pacifica invasione di turisti sbarcati dalle navi traghetto e dagli aerei giornalieri [...]. Molti sono i lampedusani che apertamente dichiarano la loro avversione alla valorizzazione turistica dell'isola, che si vorrebbe addirittura scoraggiare con ogni mezzo. Sono in massima parte gli anziani i quali tuttavia, pur degni di comprensione per i legittimi timori di una "contaminazione" della quiete e delle bellezze naturali di cui sono giustamente gelosi, non propongono soluzioni alternative per il progresso economico dell'isola (Mancini 1979: 74).

Oltre agli investimenti della gente del posto, molte iniziative e risorse economiche per lo sviluppo del turismo giunsero negli anni ' 80 -'90 anche da "nuovi lampedusani", ossia da coloro i quali, arrivati per la prima volta sull'isola come turisti, decisero di trasferirvisi, come abbiamo visto nel caso della toscana Annalisa e del borgo dei dammusi di Cala Creta. Una concorrenza che non di rado ha generato tensioni e sedimentato stereotipi reciproci, come riconosce Laura, nata a Verona, sposata con un pescatore lampedusano e qui residente ormai da oltre vent'anni:

Chi è nato qui tende sempre a vederci come forestieri, venuti a colonizzare l'isola e a rubare le ricchezze che spetterebbero di diritto a loro. E del resto chi è arrivato qui innamorato dell'isola e con tutte le migliori intenzioni di valorizzarla, spesso ha l'idea che gli isolani alla fin fine siano gente ignorante, incapace di preservare le bellezze del territorio, interessati solo al soldo. I lampedusani con cui io personalmente mi trovo meglio sono quelli che potrei definire un po' degli outsider, che magari hanno vissuto qualche anno fuori dall'isola, hanno studiato fuori e poi quando ritornano si ritrovano a essere un po' marginali ed eccentrici rispetto alla stessa comunità locale.

Vi è stato anche chi ha contribuito allo sviluppo turistico di Lampedusa giungendovi da ben più lontano e ben prima dell'inizio dei flussi di migrazione internazionale che hanno coinvolto l'isola a partire dagli anni Duemila. È questo il caso di Sami, originario del Pakistan, che nel 1987 ottenne la prima licenza per la vendita ambulante sulle spiagge di Lampedusa, e che oggi è proprietario di un bel negozio su via Roma. Grazie alla sua intraprendenza, la tartaruga di mare è diventata uno dei simboli turistici di Lampedusa, contribuendo in modo sostanziale al processo di branding dell'isola. I souvenirs di Sami, apparentemente frutto di una tradizione artigianale locale, sono in realtà l'esito di un'originale filiera creativa e produttiva di tipo transnazionale:

Ho pensato che dovevo introdurre qualcosa di nuovo, tanti turisti venivano qui per vedere le tartarughe, così prima mi sono rivolto a un ceramista in Umbria che avevo conosciuto qui come turista... poi dopo un po' ho deciso che poteva diventare un'attività in cui coinvolgere le persone che conoscevo giù in Pakistan, nella 
regione da dove vengo ci sono molti artigiani e così ora tutto quello che vedi qui nel mio negozio con il disegno della tartaruga viene dal Pakistan, dove ho ceramisti, artisti e calligrafi che lavorano per me. Vedi, su tutte le tartatughine di ceramica ho fatto scrivere: Lampedusa - Made in Pakistan. i loro frutti: nel 2008 si contavano 75 strutture ricettive (41 hotel e 34 tra campeggi, case vacanze e affittacamere) per oltre 1.900 posti-letto disponibili (senza contare le numerosissime case e stanze affittate in nero), e si superarono a fine anno i 128 mila arrivi sull'isola (circa 95 mila passeggeri giunti in aereo, 25 mila in traghetto e 8 mila in aliscafo), il 34\% in più rispetto all'inizio del decennio (Cosenza 2011).

\section{Nostra Signora delle Coperte Isotermiche}

I dati presentati in chiusura del precedente paragrafo si riferiscono al 2008 poiché quell'anno segnò il passaggio a una nuova fase della storia di mobilità dell'isola. Se infatti fin dalla fine degli anni ' 90 erano attestati arrivi via mare di migranti sull'isola, è con la costruzione del grande CSPA (Centro per il Soccorso e la Prima Accoglienza) in contrada Imbriacola nel 2007, e con la successiva crisi dell'inverno 2008-2009 dovuta al blocco dei trasferimenti dei migranti arrivati sull'isola, che Lampedusa diventa ufficialmente nel discorso pubblico, politico e mediatico "l'isola degli sbarchi" (Gatta 2012).

Su quanto accaduto sull'isola in termini di accoglienza e respingimenti, soprattutto in concomitanza con la cosiddetta "Emergenza Nord Africa" del 2011 (anno in cui giunsero a Lampedusa oltre 50 mila migranti), è stato scritto moltissimo, sia in termini di ricerca accademica che di reportage giornalistici (Sanfilippo, Sciajola 2010, Nicolosi 2016, Aime 2018, Proglio, Odasso 2018). Io intendo qui concentrarmi su quanto questo ultimo decennio abbia inciso sui processi di patrimonializzazione dell'isola, in particolare dal momento in cui, con l'elezione a sindaco di Giusi Nicolini (nel 2012) e con la visita papale di Jorge Bergoglio (luglio 2013), il discorso sull'immigrazione è stato volto a sostegno di un immaginario legato allo spirito accogliente, solidale e caritatevole dell'isola.

31 Faccio di seguito riferimento a un caso specifico di ri-signficazione del patrimonio culturale inerente la sfera del sacro e le pratiche rituali legate alla devozione per la Madonna di Porto Salvo, patrona di Lampedusa. Il culto mariano è connesso a una specifica località del versante meridionale dell'isola, oggi conosciuta col nome di Cala Madonna. In questa insenatura caratterizzata da un profondo vallone su cui si aprono alcune grotte, numerose fonti storiche risalenti a diversi secoli prima della colonizzazione borbonica attestano come esistesse un sito sacro sia per i naviganti musulmani che per i cristiani, che vi deponevano egualmente offerte e vi si rifugiavano in caso di necessità (Taranto 2015). Come scrisse Bernardo Sanvisente nella sua relazione a Ferdinando II di Borbone del 1847:

Nel Vallon de la Madonna eravi una chiesetta con antichi abituri, una casa diruta e diverse grotte. Nella chiesetta, che io trovai in meschino stato, eravi una statua di una Vergine mutilata e gettata al suolo; la quale io feci restaurare e disposi che ogni 22 settembre si cantasse una messa onde solennizzare il giorno della restaurazione stessa e del possesso dell'isola [...]. La su indicata chiesetta serviva dapprima a doppio uso, siccome al mio giungere sull'isola osservai ancora [...]. Questo locale serviva per gli arabi che transitavano per qua e che bramavano fare le orazioni di

Archivio antropologico mediterraneo, Anno XXII, n. 21 (1) | 2019 
loro religione. Più a fondo, aperto il cancello, si presentava un secondo locale ove i fedeli che desideravano visitare la miracolosa immagine trovavano l'altare cristiano con sopra la Santa Vergine già mentovata (cit. in Taranto 2015: 14).

Dal momento della colonizzazione le celebrazioni per la Madonna di Porto Salvo divennero dunque il momento centrale del calendario religioso e civile dell'isola, che in quell'occasione festeggia il culto di Maria e al tempo stesso la fondazione della sua comunità portando in processione la statua della Madonna per le vie del paese. La Madonna è invocata innanzitutto come protettrice dei pescatori e dei marinai, che non mancano mai di affiggere una sua immagine sulle proprie imbarcazioni. Tradizionalmente l'intera popolazione isolana partecipa alla processione del 22 settembre, e anche coloro i quali vivono lontani dall'isola vi fanno ritorno per trovare i parenti e mantenere i legami con la comunità.

È interessante notare come ai significati locali di questa devozione mariana, negli ultimi anni siano andati sovrapponendosene altri di scala globale, connessi al ruolo che Lampedusa gioca nel quadro delle attuali migrazioni mediterranee e alla narrazione di Lampedusa come "isola dell'accoglienza" (Melotti et al. 2018). Un ruolo cruciale in questo processo è stato sicuramente svolto dalla visita che Papa Francesco ha compiuto a Lampedusa l'8 luglio del 2013. La presenza di Bergoglio sull'isola, accompagnata da uno straordinario interesse mediatico, ha segnato l'avvio di un pontificato che ha fatto proprio del "Vangelo dell'accoglienza" la caratteristica distintiva a favore di un chiaro posizionamento della chiesa cattolica rispetto al tema delle migrazioni (Bergoglio 2017). In occasione della sua omelia lampedusana, Papa Francesco così invocò la Madonna di Porto Salvo:

Maria, Madre di Dio e Madre nostra, volgi il tuo sguardo dolcissimo su tutti coloro che ogni giorno affrontano i pericoli del mare, per garantire alle proprie famiglie il sostentamento necessario alla vita, per tutelare il rispetto del creato, per servire la pace tra i popoli. Protettrice dei migranti e degli itineranti, assisti con cura materna gli uomini, le donne e i bambini costretti a fuggire dalle loro terre in cerca di avvenire e di speranza.

Da quel momento in avanti, un'ampia iconografia atta a esprimere la nuova raffigurazione della "Madonna dei migranti" è stata introdotta in diversi spazi del sacro a Lampedusa, e in particolare presso la chiesa parrocchiale e il santuario di Cala Madonna, recuperando simbolicamente e valorizzando i riferimenti all'isola come luogo di incontro interreligioso e "porto salvo" per cristiani e musulmani.

In questa prospettiva si può leggere un significativo passaggio dell'omelia con cui il 22 settembre 2018 il vescovo di Cefalù, Giuseppe Marciante, ha introdotto la Solenne Celebrazione Eucaristica per la Festa della Madonna di Porto Salvo in una chiesa gremita di autorità, turisti e cittadini lampedusani:

Lampedusa è l'incarnazione della Madonna di Porto Salvo, un'isola-grembo che come una madre accoglie umanità e genera figli. Lampedusa è una terra che ha sempre accolto le genti: maltesi, greci, spagnoli, così come Maria ha sempre accolto le preghiere che gli giungevano dai suoi figli, italiani, arabi, cristiani e musulmani [...]. Sono venuto qui sulle orme di Papa Francesco, per incontrare i migranti e la devozione della comunità lampedusana per Maria. Era un mio sogno venire a Lampedusa, perché quest'isola per me è un simbolo, un segno di umanità. Questa è l'isola dell'accoglienza, un'accoglienza di persone che provengono da ogni parte del mondo e che è benedetta da Dio. Un'accoglienza su cui si fonda anche il vostro benessere, visto che tante altre persone arrivano sull'isola attratte da questo mare bellissimo, che è anch'esso una benedizione di Dio, e anch'io questa mattina ho avuto piacere di fare un giro dell'isola accompagnato da don Carmelo e ho 
ammirato la bellezza di questa terra [...]. Al molo ho incontrato un gruppo di migranti, eritrei cristiani e tunisini musulmani, e ho promesso che avrei portato a Maria le loro preghiere e le loro invocazioni. Lo faccio qui ora: Maria, proteggili e accompagnali nel loro viaggio di speranza! Madonna di Porto Salvo si è in qualche modo estesa giungendo a inglobare una nuova data carica di un duplice valore civile e spirituale: il 3 ottobre infatti, giorno in cui avvenne il tragico naufragio in cui persero la vita quasi 400 migranti, è divenuta ufficialmente "Giornata nazionale in memoria delle vittime dell'immigrazione", istituita dal Parlamento italiano nel 2015 e celebrata da quel momento in avanti con la presenza sull'isola delle massime cariche dello Stato e dei rappresentanti dei sopravvissuti al naufragio e di alcuni familiari delle vittime (Vietti 2018).

Tra i luoghi coinvolti da questa nuova ricorrenza vi è anche il Santuario della Madonna di Porto Salvo. Qui, il 3 ottobre 2018, l'artista fiorentino Giovanni De Gara, in accordo con il parroco di Lampedusa Carmelo La Magra, ha ricoperto la porta d'accesso alla chiesa con i teli termici dorati che vengono forniti ai migranti durante i salvataggi in mare, a rappresentare simbolicamente come per i fedeli l'incontro con Maria possa avvenire solo attraversando la soglia dell'incontro con la sofferenza dell'altro. Un diverso messaggio, ma un simile ricorso simbolico alla superficie dorata dei teli termici, lo si ritrova anche nell'opera che l'attivista lampedusano Giacomo Sferlazzo ha intitolato "Nostra Signora delle Coperte Isotermiche": una statuetta in legno della Madonna ritrovata su una barca di migranti naufragata sull'isola, che, avvolta in una coperta isotermica, è stata donata alla parrocchia di Lampedusa per essere collocata nell'area delle grotte dell'antico eremitaggio di Cala Madonna, presso il Santuario. Accompagna questa trasfigurazione della Madonna di Porto Salvo una preghiera laica che ne esplicita la valenza politica:

Nostra Signora delle Coperte Isotermiche / aiutaci a salvare coloro che non riusciremo ad uccidere bombardando / e quando li rinchiuderemo nei centri di detenzione / facceli accudire come il Dio degli eserciti comanda [...].

Un oggetto sacro dunque che denuncia e mira a sovvertire le logiche dominanti del securitario e dell' umanitario connesse alla gestione delle migrazioni e alla militarizzazione di Lampedusa e del Mediterraneo (Fassin 2018).

\section{Conclusioni}

Ogni anno Lampedusa batte i suoi record in fatto di arrivi turistici: 185 mila persone atterrate sull'isola nel 2015, 225 mila nel 2016, oltre 250 mila nel 2017 (Enac 2018). Amministratori e albergatori locali celebrano percentuali di crescita straordinaria e preannunciano il "tutto completo" per luglio e agosto. Su Tripadvisor gli utenti mettono in guardia dalla "folla" che si incontra sulle spiagge e per le strade dell'isola, consigliando di visitarla "fuori stagione". Una stagione che, se un tempo si concludeva a inizio settembre, ora si è estesa fino ad autunno inoltrato: la collaborazione che l'amministrazione comunale di Lampedusa ai tempi di Giusi Nicolini ha avviato con l'Associazione Italiana Turismo Responsabile (AITR), ha portato a Lampedusa un numero crescente di turisti interessati a partecipare alle tradizioni del luogo, come nel caso della Festa della Madonna di Porto Salvo (Rossi 2014). Per la commemorazione del naufragio del 3 ottobre giungono inoltre sull'isola migliaia di persone tra delegazioni, migranti, giornalisti, forze dell'ordine, rappresentanti di organizzazioni non 
governative e associazioni che necessitano, in modo non dissimile dai turisti, di hotel dove soggiornare e ristoranti in cui mangiare.

Che cosa vedono queste persone mentre sfilano per le strade di Lampedusa, cosa cercano nelle poche ore o nei giorni in cui si trattengono sull'isola? Vorrei qui tornare ancora una volta sulla spiaggia della Guitgia e alla fotografia con cui ho iniziato questo contributo. Quell'incrocio di sguardi mi sembra ben evocare le stratificazioni di immaginari che hanno plasmato nel corso del tempo il patrimonio di Lampedusa: migranti e turisti sono tutti a loro modo outsiders, "nomadi della contemporaneità" che si muovono in un mondo in cui la mobilità ha perso la sua caratteristica di eccezionalità per diventare elemento costitutivo della produzione culturale e identitaria (Callari Galli 2004).

41 Come hanno scritto Vincenzo Padiglione e Alessandra Broccolini (2017), l'emergere in Italia di "comunità patrimoniali" che si consolidano attorno a un "nuovo ordine di differenze culturali" apre per gli antropologi la necessità di un riflessione sulle modalità e sul grado del loro coinvolgimento:

Quando il patrimonio diviene un nucleo di poetiche sociali, la matrice di politiche culturali, il focus di iniziative commerciali, la stella cometa di molta immaginazione in sintonia con lo spirito del tempo, il fare comunità riguarda (e in che modo?) anche lo studioso? [...] È evidente che in funzione della risposta si dischiudono poteri contrattuali e spazi di partecipazione critica differenti (Padiglione, Broccolini 2017: 5).

In questa prospettiva, non stupisce che numerosi antropologi abbiano fatto di Lampedusa il loro campo non solo di studio, ma anche di applicazione del proprio sapere attraverso progetti nati dal loro peculiare regard éloigné, lo "sguardo da lontano" di coloro che per professione si pongono sulla soglia tra interno ed esterno della comunità locale (Lévi-Strauss 1984). Emblematico di questo impegno è il lavoro svolto dall'Archivio delle Memorie Migranti (AMM) di Roma, che ha sviluppato un ampio corpus di produzioni audio-video dedicate proprio ad esplorare le diverse narrazioni dell'isola da parte dei residenti e dei migranti. Migranti giunti a Lampedusa dal Corno d'Africa, come nel caso di Dagmawi Yimer, Mahamed Aman e Zakaria Mohamed Ali, che AMM ha accompagnato in esperienze di ritorno sull'isola documentando il loro incontro con i luoghi e le persone che li avevano visti sbarcare come "clandestini" e che li vedono tornare dopo qualche anno come testimoni e, secondo la loro stessa autorappresentazione, come "turisti" (Horsti 2019).

43 Condividendo un simile approccio, anch'io ho ritenuto di poter intrecciare la mia riflessione critica sul nesso tra mobilità e patrimonio nel Mediterraneo prima con l'attività di ideazione e accompagnamento di esperienze di turismo responsabile a Lampedusa e poi con una collaborazione didattica con l'Istituto superiore turisticoalberghiero E. Majorana, nato nel 2014 per preparare i giovani isolani a entrare in modo professionale nel settore trainante dell'economia locale.

Ed è stato proprio lo sguardo di questi studenti ad avermi evocato un ultimo, ulteriore immaginario prodotto dalla connessione tra migrazioni e turismo. Nelle parole di Niccolò, 17 anni, l'esperienza degli "africani" che tutti vengono a cercare a Lampedusa diventa uno specchio che riflette le proprie paure e i propri desideri di fuga, facendo del mondo "fuori" dall'isola un'eterotopia dove proiettare la propria aspirazione alla felicità:

Io a dir la verità sono stufo di parlare sempre degli africani... a scuola viene sempre qualcuno a proporci di fare cose sugli immigrati, concorsi letterari, gemellaggi con 
altre scuole, interviste. Non che non sia una cosa interessante, ma io devo pensare al mio futuro, o riesco a fare qualcosa qui con i turisti, oppure me ne andrò fuori... io ho sempre sognato di vivere a Londra, o almeno a Milano. proprio futuro.

\section{BIBLIOGRAFIA}

Adey P., 2017 Mobility, Routledge, New York.

Aime M., 2005 L'incontro mancato, Bollati Boringhieri, Torino.

Aime M., 2018 L'isola del non arrivo. Voci da Lampedusa, Bollati Boringhieri, Milano.

Appadurai A., 2001 Modernità in polvere, Meltemi, Roma.

Bergoglio J.M., 2017 La sfida dei migranti. Scritti, discorsi e omelie, EDB, Bologna.

Callari Galli M. (a cura di), 2004 Nomadismi contemporanei. Rapporti tra comunità locali, stati-nazione e 'flussi culturali globali', Guaraldi, Rimini.

Callari Galli M., Harrison G., 1972 «Situational Signs and Social Attentiveness: The Conception of Reality Among a Group of Sicilian Illetterates», in M.D Loflin, J. Silverberg (eds), Discourse and Inference in Cognitive Anthropology. An Approach to Psychic Unity and Enculturation, Mouton, The Hague: $167-198$.

Clifford J., 2008 I frutti puri impazziscono. Etnografia, letteratura e arte nel secolo XX, Meltemi, Roma.

Cosenza G., 2011 «I contrasti delle Pelagie: fra turismo di massa, ambientalismo e migranti», in P. Violi, A.M Lorusso (a cura di), Effetto Med. Immagini, discorsi, luoghi, Lupetti, Bologna: 283-319.

Cuttitta P., 2012 Lo spettacolo del confine. Lampedusa tra produzione e messa in scena della frontiera, Mimesis, Milano.

Enac, 2018 Dati di traffico 2017, www.enac.gov.it/sites/default/files/allegati/

2018-Ago/Dati_di_Traffico_2017_it.pdf (ultimo accesso: 16 aprile 2019).

Fassin D., 2018 Ragione umanitaria. Una storia morale del presente, DeriveApprodi, Roma.

Fragapane G., 1993 Lampedusa, Sellerio, Palermo.

Gatta G., 2012 «Corpi di frontiera. Etnografia del trattamento dei migranti al loro arrivo a Lampedusa», in AM. Rivista della Società italiana di antropologia medica, 33-34: 129-161.

Gatta G., Muzzopappa G., 2012 «'Middle Passages’, musealizzazione e soggettività a Bristol e Lampedusa», in Estetica. Studi e ricerche, 1: 167-81.

Glick Schiller N., Salazar N., 2013 «Regimes of Mobility across the Globe», in Journal of Ethnic and Migration Studies, 39, 2: 183-200.

Hall C.M., Williams A.M. (eds), 2002 Tourism and Migration. New Relationships between Production and Consumption, Kluwer, Dordrecht. 
Horsti K., 2019 «Temporality in cosmopolitan solidarity: Archival activism and participatory documentary film as mediated witnessing of suffering at Europe's borders", in European Journal of Cultural Studies, 22, 2: 231-244.

Isola A., 2018 Capperi! Dal 2011, la guida a godersi il meglio di Lampedusa, Linosa, Lampione, I-Copy Textplayers, Lampedusa.

Lauth Bacs J., 2013 «Managing Proximity and Asymmetry in Border Encounters: The Reception of Undocumented Migrants on a Greek Border Island», in J. Lauth Bacas, W. Kavanagh (eds), Border Encounters - Asymmetry and Proximity at Europe's Frontiers, Berghahn Books, New York: 255-280.

Lauth Bacas J., Kavanagh W (eds), 2013 Border Encounters - Asymmetry and Proximity at Europe's Frontiers, Berghahn Books, New York.

Lenz R., 2010 «'Hotel Royal' and other Spaces of Hospitality: Tourists and Migrants in the Mediterranean», in J. Scott, T. Selwyn (eds), Thinking Through Tourism, New York, Berg: 209-230.

Lévi-Strauss C., 1984 Lo sguardo da lontano, Einaudi, Torino.

Mazzara F., 2015 «Spaces of Visibility for the Migrants of Lampedusa: The Counter Narrative of the Aesthetic Discourse», in Italian Studies, 70, 4: 449-64.

Mazzara F., 2016 «Subverting the Narrative of the Lampedusa Borderscape», in Crossings: Journal of Migration \& Culture, 7, 2: 135-47.

Melotti M., Ruspini E., Marra E., 2018 «Migration, Tourism and Peace: Lampedusa as a Social Laboratory», in Anatolia. An International Journal of Tourism and Hospitality Research, 29, 2: 215-224.

Nicolosi G., 2016 Lampedusa. Corpi, immagini e narrazioni dell'immigrazione, Franco Angeli, Milano. Orsini G., 2015 «Lampedusa: From a Fishing Island in the Middle of the Mediterranean to a Tourist Destination in the Middle of Europe's External Border», in ItalianStudies, 70, 4: 521-536.

Padiglione V., Broccolini A., 2017 «"Uscirne insieme”. Farsi comunità patrimoniale», in AM Antropologia Museale, 13, 37-39: 3-10.

Proglio G., Odasso L. (a cura di), 2018 Border Lampedusa. Subjectivity, Visibility and Memory in Stories of Sea and Land, Palgrave Macmillan, Basingstoke.

Riccio B. (a cura di), 2019 Mobilità. Incursioni etnografiche, Mondadori, Milano.

Roghi G., 2013 «Lampedusa. L'isola degli sgombri», in Quaderni dell'Associazione Culturale Archivio Storico di Lampedusa, 2: 1-9.

Rossi I., 2014 Lampedusa. Guida per un turismo umano e responsabile, Altra Economia, Milano.

Sanfilippo F., Sciajola A., 2010 A Lampedusa. Affari, malaffari, rivolta e sconfitta dell'isola che voleva diventare la porta d'Europa, Infinito edizioni, Roma.

Taranto A., 2013 Breve storia di Lampedusa, Associazione Culturale Archivio Storico di Lampedusa, Lampedusa.

Taranto A., 2015 Storia del Santuario della Madonna di Porto Salvo a Lampedusa, Associazione Culturale Archivio Storico di Lampedusa, Lampedusa.

Urry J., 2000 Sociology Beyond Societies: Mobilities for the Twenty-First Century, Routledge, London.

Vietti F., 2018 «Mare Morto. Tracce ed evocazioni dei missing migrants a Lampedusa», in Studi Tanatologici, 9: 41-67. 


\section{NOTE}

1. Nel dettaglio: ho svolto il ruolo di accompagnatore turistico per quattro edizioni del programma di viaggio "Lampedusa, crocevia del Mediterraneo" organizzato dal tour operator di turismo responsabile "Viaggi Solidali" di Torino (2014-2017); sono stato tutor del progetto alternanza-scuola lavoro per gli studenti dell'Istituto Omnicomprensivo Luigi Pirandello di Lampedusa nell'ambito della VIII Scuola di Sociologia del Territorio (2018); ho compiuto ricerca di campo grazie all'assegno di ricerca "Comunità patrimoniali e ruolo pubblico dell'antropologia" dell'Università di Milano Bicocca (2018-2019).

2. Ringrazio per la disponibilità e l'aiuto Antonino Taranto dell'Archivio Storico di Lampedusa; Giacomo Sferlazzo e Francesca Del Volgo del collettivo Askavusa; Elena Prazzi di Legambiente; lo staff di Meditrerranean Hope - Federazione delle chiese evangeliche in Italia; Paola Pizzicori e tutti i soci del Forum Lampedusa Solidale.

3. Il mio approccio alla storia dell'isola è dichiaratamente parziale e tematico. Per un'analisi approfondita della storia generale di Lampedusa, incluso l'interessante e lungo periodo precedente la colonizzazione borbonica, si veda il ben documentato volume di Giovanni Fragapane (1993).

4. L'attività politica, sociale e artistica di Giacomo Sferlazzo e del collettivo Askavusa è stata oggetto di diverse ricerche antropologiche e non solo, cui qui si rimanda per un approfondimento: Gatta, Muzzopappa (2012), Mazzara (2015, 2016).

\section{RIASSUNTI}

Gli studi sulla mobilità possono fornire un utile approccio per interpretare le connessioni tra migrazioni e turismo che caratterizzano numerosi contesti mediterranei. Alcune località insulari che fondano la propria sussistenza economica sull'attrattività turistica, negli ultimi anni sono infatti divenute luogo di approdo e transito di significativi flussi migratori. Il contributo si concentra sul caso di Lampedusa, attraverso una ricerca etnografica volta a indagare il ruolo svolto dalla mobilità nelle successive fasi storiche di trasformazione sociale, culturale ed economica dell'isola. Studiando i processi di patrimonializzazione in atto, con particolare riferimento al locale culto della Madonna di Porto Salvo, si intende mettere in luce le modalità attraverso cui le migrazioni sono state integrate nel patrimonio culturale di Lampedusa e sono divenute parte costitutiva della sua immagine turistica.

Mobility studies can provide a fruitful approach to understand the relationship between migration and tourism in many Mediterranean contexts. Some islands that rely on tourism for their economic survival have in recent years become a place of transit and reception of significant migration flows. This contribution focuses on the case of Lampedusa, through an ethnographic research aimed at investigating the role played by mobility in the subsequent historical periods of social, cultural and economic transformation of the island. By studying the processes of heritage-making underway, with particular reference to the local cult of Our Lady of Porto Salvo, the aim is to highlight the ways in which migrations have been integrated into the cultural heritage of Lampedusa and have become a constitutive part of its tourist image. 
INDICE

Keywords : tourism, migration, heritage, mobility, Mediterranean, Lampedusa

Parole chiave : turismo, migrazione, patrimonio, mobilità, Mediterraneo, Lampedusa

\author{
AUTORE \\ FRANCESCO VIETTI \\ Dipartimento di Scienze Umane per la Formazione "Riccardo Massa", Università degli Studi di \\ Milano Bicocca francesco.vietti@unimib.it
}

